\title{
Mesure de la dynamique couplée de propagation et d'ionisation d'une impulsion laser - Application au laser X-OFI ou à la génération d'harmoniques d'ordres élevés
}

\author{
C. Valentin, F. Weihe, S. Kazamias, R. Haroutunian, S. Sebban, G. Grillon, \\ F. Augé, G. Chériaux, A. Rousse, D. Hulin et Ph. Balcou \\ Laboratoire d'Optique Appliquée, Chemin de la Hunière, ENSTA, École Polytechnique, \\ UMR 7639 du CNRS, 91761 Palaiseau cedex, France
}

\begin{abstract}
Résumé : Nous avons utilisé une technique récente de mesure d'impulsions ultracourtes afin d'étudier la dynamique de l'ionisation et la propagation dans un milieu gazeux. Nous présentons les mesures de variation de l'intensité, de la phase temporelle et du spectre d'une impulsion laser intense $\left(10^{16} \mathrm{~W} / \mathrm{cm}^{2}\right)$, ultracourte $(35 \mathrm{fs}$ ) après son interaction avec un milieu gazeux. Les variations de l'indice de réfraction du plasma sont rapides et modifient la structure spatiale et temporelle de limpulsion lors de sa propagation dans le gaz. Ces variations d'intensité et de phase temporelles peuvent être utrilisées pour connaitre la dynamique de l'interaction laser-plasma. En particulier, cette expérience permet de mettre en évidence les effets sur la génération d'un milieu de gain pour le laser $\mathrm{X}$ de type OFI (Optical Field Ionization).
\end{abstract}

\section{Introduction}

Le développement de lasers ultracourts de haute intensité a rendu possible l'étude de nouveaux processus [1]. Beaucoup d'entre eux, tels que la génération d'harmoniques d'ordres élevés $[2,3,4]$ et la génération de milieu de gain pour le laser X-OFI [5], dépendent de façon critique de la forme de l'impulsion laser, résultant de la dynamique de propagation d'une impulsion laser intense traversant un gaz de faible pression $(\mathrm{P} \sim 10$ à 20 torr) à des intensités de focalisation variant de $10^{14}$ à $10^{16} \mathrm{~W} / \mathrm{cm}^{2}$. On rappelle que pour des intensités de quelques $10^{16} \mathrm{~W} / \mathrm{cm}^{2}$, on s'attend à atteindre un degré d'ionisation suffisamment élevé pour la génération do gain pour le laser $\mathrm{X}$ de type OFI.

Dans une première partie, nous présentons une étude expérimentale de la dynamique de propagation d'une impulsion intense et ultracourte traversant un milieu gazeux non linéaire. Notre technique de mesure nous permet de mesurer des effets à une échelle de temps plus petite que la largeur temporelle de l'impulsion ( $35 \mathrm{fs}$ ). Dans un deuxième temps, nous discutons des processus non linéaires qui mènent alıx formes d'impulsion observées et présentons les résultats de simulations numériques. Enfin, nous concherons sur l'intérêt de cette étude pour les expériences de laser X-OFI.

\section{Expérience et résultats expérimentaux}

Le système laser utilisé est un Ti:Sa de cadence $1 \mathrm{kHz}$ produisant des impulsions de polarisation linéaire, de largeur à mi-hauteur $35 \mathrm{fs}$ et d'énergie $7 \mathrm{mIJ}$ avec une longueur d'onde centrale de $805 \mathrm{um}$ et un faiscean de largeur à $1 /$ e de $22 \mathrm{~mm}$. Le faisceau laser est focalisé sous vide par une lentille de focale $750 \mathrm{~mm}$ dans une cellule remplie de gaz rare. La pression du gaz dans la cellule peut varier de 0 à 30 torr. Les intensités focalisées sont do l'ordre de $10^{16} \mathrm{~W} / \mathrm{cm}^{2}$ dans le vide. Dans la cellule, ces intensités sont plus faibles que dans le cas gaussien idéal à cause 
des effets de propagation sur la forme spatiale et temporelle de l'impulsion et des imperfections du front d'onde. Dans cette expérience, les mesures ont été réalisées avec une cellule d'argon de $1 \mathrm{~mm}$. Le faisceau divergent issu de la cellule est collimaté par une deuxième lentille de focale $750 \mathrm{~mm}$. Une partie de ce faisceau est dirigée vers notre système de mesure, basée sur la technique SPIDER (Spectral Phase Interferometry for Direct Electric-field Reconstruction) [6]. La résolution temporelle est de quelques fs, limitée par la largeur spectrale cohérente du laser après l'interaction. Nous avons enregistré l'intensité $\mathbf{I}(\mathbf{t})$ et la phase temporelle $\phi(t)$. Le SPIDER permet aussi de mesurer indépendamment le spectre I $(\omega)$ de l'impulsion à l'aide d'un spectromètre.

Nous avons observé des variations de la forme de l'impulsion $\mathrm{I}(\mathrm{t})$ et de la phase temporelle $\phi(t)$ en fonction de la pression de gaz. La figure (1a) montre les variations de $\mathrm{I}(\mathrm{t})$ pour une pression nulle et une pression de 30 torr. Pour cette expérience, le foyer est en entrée de cellule. Dans ces conditions, l'intensité crête est importante et les interactions non linéaires entre le laser et le milieu sont très fortes. La propagation d'une impulsion laser intense à travers un plasma en cours de formation résulte du couplage complexe entre les phénomènes de propagation et d'ionisation. Lorsqu'elle entre dans la cible gazeuse, l'impulsion induit une ionisation partielle du milieu sur une échelle de temps ultracourte. L'enveloppe de l'impulsion à la sortie de la cellule est fortement modulée avec des épaulements sur le front montant dus à l'ionisation et une post-impulsion conséquente. Nous pensons, d'après le spectre mesuré, que cette post-impulsion provient de la diffusion Raman Stoke et anti-Stoke induite par la propagation complexe du laser dans le plasma en formation [7]. En effet, le spectre présente deux sous-structures en bandes latérales situées à $20 \mathrm{~nm}$ de la longueur d'onde centrale du laser, correspondant à la pulsation plasma calculée à 30 torr.
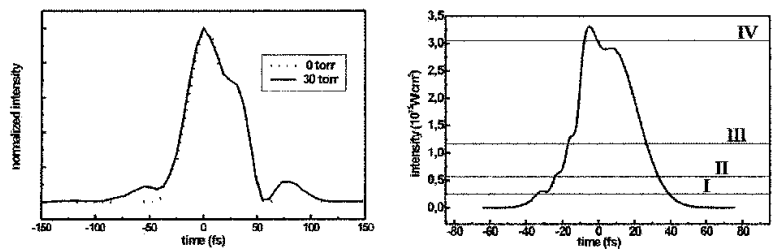

Fig. 1. Intensité temporelle mesurée (a) et calculée (b) d'une impulsion de $6.6 \mathrm{~m} J$ focalisée à l'entrée d'une cellule de gaz de $1 \mathrm{~mm}$, à 0 et 30 torr d'argon. Les lignes horizontales en (a) correspondent aux quatre premiers seuils d'intensité de suppression de barrière (BSI), marqués par des chiffres romains.

Les modulations de l'enveloppe de l'impulsion résultent de séries d'ionisations successives dans le milieu, induites par le laser. Lorsque l'intensité dans le gaz augmente, et dépasse les seuils successifs d'ionisation [8], les gradients longitudinal et transverse de l'indice de réfraction sont plus importants au début de chaque processus d'ionisation. Les variations temporelles de la phase spatiale modifient la répartition d'énergie de l'impulsion et induisent des chutes locales de l'intensité instantanée. Nous avons réalisé des simulations numériques de propagation d'une impulsion courte dans un milieu non linéaire [9] qui reproduisent qualitativement le comportement de l'impulsion dans un milieu en cours d'ionisation . 


\section{Résultats de simulation numérique}

Le code de simulation numérique repose sur la symétrie radiale naturelle du faisceau. Il est basé sur la résolution des équations de Maxwell paraxiales dans le cadre de l'enveloppe lenternent variable (SVEA). Nous avons ainsi accès non seulement à l'enveloppe de l'impulsion mais aussi à sa phase spatio-temporelle $\phi(\mathrm{r}, \mathrm{z}, \mathrm{t})$. La variation d'indice résulte de la contribution des électrons libres du plasma en formation. Notre code inclut également l'effet Kerr. L'ionisation est calculée à partir des taux d'ionisation tunnel ADK [10].

Pour des intensités telles que l'on peut négliger les effets relativistes, on peut écrire l'indice de réfraction local n du milieu, pour chaque point d'espace et de temps par :

$$
n(\omega)=\left(1-P_{I}\right)\left[n_{0}(\omega)+\operatorname{In}_{2}(\omega)\right]-\frac{e^{2} \mathcal{N}_{a} P_{I}}{2 \epsilon_{0} m \omega^{2}}
$$

où $P_{I}$ est la probabilté d'ionisation intégrée en temps, $\mathcal{N}_{a}$ est la densité du gaz, $n_{0}$ est l'indice de réfraction du milieu neutre, $n_{2}$ est l'indice non linéaire associé à l'effet Kerr [11], e est la charge de l'électron, $\epsilon_{0}$ est la permitivité du vide et $\omega$ est la pulsation du laser. Contrairement aux atomes neutres, l'indice de réfraction des ions est très proche de 1 même en tenant compte de l'effet Kerr. En appliquant des valeurs numériques à l'équation ci-dessus, le terme important dans la variation d'indice est la dispersion élcctronique dès que l'ionisation atteint quelques \% $[9]$.

La résolution temporelle du code est plus grande que la résolution expérimentale accessible par le SPIDER. C'est pourquoi nous avons résolu une série d'épaulements sur le front montant du profil d'intensité de l'impulsion (figure 1b). Ces structures sont associées à chaque étage d'ionisation lorsque l'intensité atteint le seuil d'intensité de suppression de barrière (BSI) pour chaque ionisation. Ce seuil BSI correspond à l'intensité qui doit être fournie pour ioniser chaque étage successif. Par ailleurs, une augmentation du nombre d'électrons libres diminue l'indice de réfraction. On observe des effets sur l'axe car différentes parties de l'impulsion subissent différents chemins optiques. Des effets transverses sont également visibles car l'effet de lentille divergente dû à l'ionisation défocalise le faisceau au niveau du front d'ionisation.

On traite numériquement le profil radial du faisceau en champ lointain par la transformée de Haenkel [12].
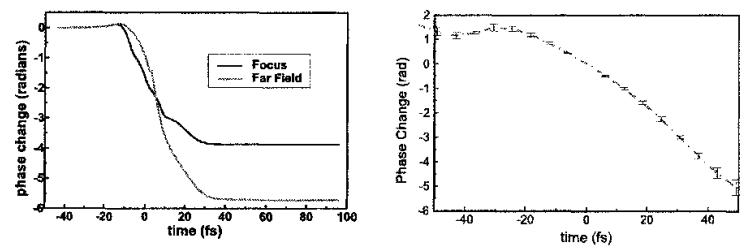

Fig. 2. Variation de la phase temporelle $\phi(t)$ calculée au point focal dans le vide dans le gaz, au foyer et en chanp lointain (a); et les variations mesurées (en champ lointain) de $\phi(t)$ pour une impulsion qui a traversée la cellule de gaz.

A cause des variations spatiales ef temporelles du chemin optique, on s'attend aussi à observer des structures étroites sur la phase temporelle $\phi(t)$, associées aux variations d'indice induites 
par chaque étape d'ionisation. Des variations plus lentes sont observées expérimentalement et numériquement (figure 2). En champ proche, nous observons bien ces structures. En champ lointain, la courbe est lissée et ces structures n'apparaissent plus expérimentalement.

\section{Applications et Conclusion}

Il est important de développer des modèles de propagation d'impulsions ultracourtes dans des cibles gazeuses afin de mieux comprendre les processus très fortement non linéaires tels que la génération d'un milieu de gain pour le laser X OFI [13]. Cette étude expérimentale nous a montré qu'il faut augmenter la puissance du laser de pompe dans le gaz. L'intensité effective permet alors d'atteindre le degré d'ionisation (en l'occurrence 8 pour le Xenon et le Krypton) requis pour la création du mileu lasant. En effet, le gain mesuré expérimentalement est important seulement si cette puissance est environ 50 fois plus élevée que celle requise pour atteindre l'intensité de seuil par suppression de barrière calculée pour ce degré d'ionisation.

Afin d'augmenter la précision du code de simulation, il serait intéressant de tenir compte des variations rapides de l'enveloppe [14]. Dans ce cas, il serait pertinent d'utiliser les taux ADK instantanés, voire des taux issus de la résolution directe de l'équation de Schrödinger prenant en compte des effets d'ionisation non-séquentielle. Ces développements n'ont pourtant jamais été réalisés pour des ionisations multiples. Les résultats de nos expériences et de nos simulations numériques représentent une amélioration dans la compréhension des phénomènes de propagation d'une impulsion intense et ultracourte dans un milieu gazeux.

\section{References}

1. Sterling Backus, Charles G. Durfee III, Margaret M. Murnane, and Henry C. Kapteyn, Rev. Sci. Inst. 69, 1207-1223 (1998).

2. A. L'Huillier, K. S. Schafer, and K. C. Kulancter, J. Phys. B 24 (1991) 3315.

3. M. Lewensteit, Ph. Balcou, M. Yu Ivanov, A. L'Huillier, and P.B. Corkum, Phys Rev. A 49 (1994) 2117.

4. S. Kazamias, F. Weihe, D. Douillet, C. Valentin, T. Planchon, S. Sebban, G. Grillon, F. Augé, D. Hulin, and Ph. Balcou, Eur. Phys. J. D 21 (2002) 353-360.

5. S. Sebban, R. Haroutunian, G. Grillon, A. Rousse, S. Kazamias, T. Marin, J.-P. Rousseau, L. Notebaert, M. Pittman, J..P. Chambaret, A.Antonetti, D. Hulin, D. Ros, A. Klisnick, A. Carillon, P.Jaeglé, G. Jamelot, and J-F. Wyart, Phys. Rev. Lett. 86 (2001) 3004-3007.

6. C. Iaconis and I. A. Walmsley, Opt. Lett. 23 (1998) 732-734.

7. W.B. Mori, C.D. Decker, D.E. Hinkel, T. Katsouleas, Phys. Rev. Lett. 72 (1994) 1482-1485.

8. S. Augst, D. Strickland, D. D. Meyerhofer, S. L. Chin, and J. H. Eberly, Phys. Rev. Lett. 63 (1989) 2212-2215.

9. R. Haroutunian, thèse de doctorat, École Polytechnique, Palaisealt, France, 2001 (non publiée).

10. M. V. Anmosov, N. B. Delone, and V. P. Krainox, Sov. Phys. JETP 64 (1986) 1191.

11. Y. R. Shen, Principles of Nonlinear Oplics, (John Wiley and Sons, 1984).

12. A. E. Siegman, Opt. Lett. 1 (1977) 13.

13. S. Sebban, T. Mocek, D. Ros, L. Upcraft, Ph. Balcou, R. Haroutunian, G. Grillon, B. Rus, A. Klisnick, A. Carillon, G. Jamelot, C. Valentin, A.Rousse, J.P. Rousseau, L. Notebaert, M. Pittman, and D. Hulin, Phys. Rev. Lett. 89 (2002) 253901 .

14. M. Geissler, G. Tempea, A. Scrinzi, M. Schnürer, F. Krausz, and T. Brabec, Phys. Rev. Lett. 83 (1999) 2930-2933. 